\title{
S-3. Nucleus Ventrales Intermedius of the Thalamus
}

\author{
Hirotaro NARABayashi and Akira Goto \\ Dept. of Neurology, Juntendo Medical School \\ Ryuichi NAKAMURA \\ Dept. of Orthopediatrics, Faculty of Medicine, University of Tokyo \\ Kisou Kubota \\ Dept. of Neurophysiology, Brain Research Institute, University of Tokyo \\ Kenji Kosaka \\ 2nd Dept. of Physiology, Faculty of Medicine, University of Tokyo \\ Tomonori NAGAO \\ Neurological Clinic, Tokyo
}

Nucl. ventralis intermed. (Vim) of the thalamus, which is lying posteriorly to VL and anteriorly to the sensory relay nuclei, was investigated and proved that in this small nucleus the lowest threshold muscle afferents are sending projection. By using CAT computer, triphasic potential could be recorded from this small area, for ulnar nerve shocks at intensities below the threshold for evoking the muscle contractions.

Velocity for this conduction was measured as $86 \mathrm{~m} / \mathrm{sec}$ ( 9 cases).

This Vim area was also experienced as mainly concerned with tremor rather than with rigidity. Electrical stimulation of Vim produces selective increase or decrease of tremor and not of rigidity. Small surgical lesion in this area results in alleviation of tremor and not of rigidity.

\section{S-4. The Effects of Electrical Stimulation on the Motor and Sensory System during Stereotaxic Operations}

\author{
Kenichiro Sugita, Terushige Dor, Toshiro Takaoka, Nomi Mutsuga, \\ Yoshiya Miura, Norihide NaKashima and Masamitsu NaKaJima \\ 1st. Surgical Dept., School of Med., Nagoya University \\ Chukyo Hospital, Nagoya and Saiseikai Hospital, Shizuoka
}

The characteristic effects of electrical stimulation in Ventro-oralis, Centre médian of thalamus and Pallidum internus on the motor and sensory function are observed during 200 streotaxic operations. In $75 \%$ of cases of V.o. posterior 
stimulation the spontaneous tremors of contralateral fingers clearly synchronize with each volley of the 5 to $9 \mathrm{cps}$ stimulation, while such forced tremors are observed only in $30 \%$ during V.o. anterior stimulation. In the patients without extrapyramidal disorder the stimulation of V.o. results in no change of the motor function or has very high threshold to cause any effect on the system. In 90\% of cases of CM stimulaItion the pain or heat sensation is induced and in $60 \%$ its stimulation evokes involuntary movements, especially typical tremor that synchronizes with frequencies of stimulation such as seen in the cases of V.o. stimulation. The stimulation of Pallidum internus does not change frequency of tremor but modifies only amplitude and muscle tone.

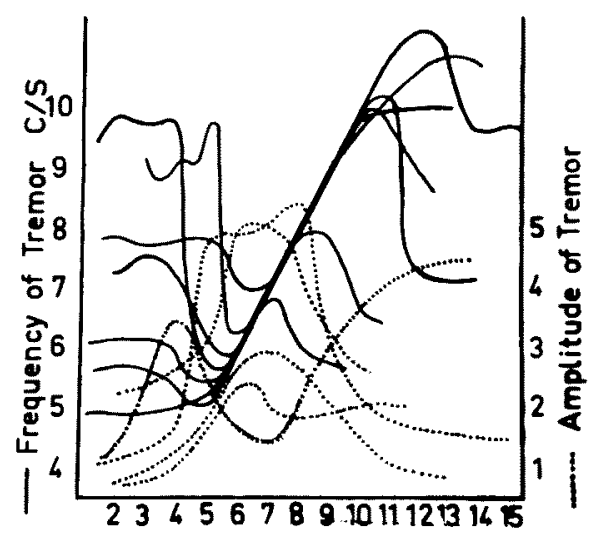

The relation between frequency and amplitude of tremor and stimulating frequency in 7 cases of V.o. and CM stimulation.

\section{S-5. The Motor Responses to the Stimulation of the Rostral Human Brain Stem}

Keiji Sano, Masumi Yoshioka, Motohide Ogashiwa, Buichi IshiJima, Chihiro Oye, Hiroaki Sekino and Yoshiaki Mayanagr

Department of Neurosurgery, Tokyo University

During streotaxic operations for the violent behavior (under general anesthesia) and spasmodic torticollis (awaked state), various types of motor responses are obtained by the high cycle stimulation of the rostral brain steam. They mostly consist of neck and ocular movements, and extremities are scarcely 